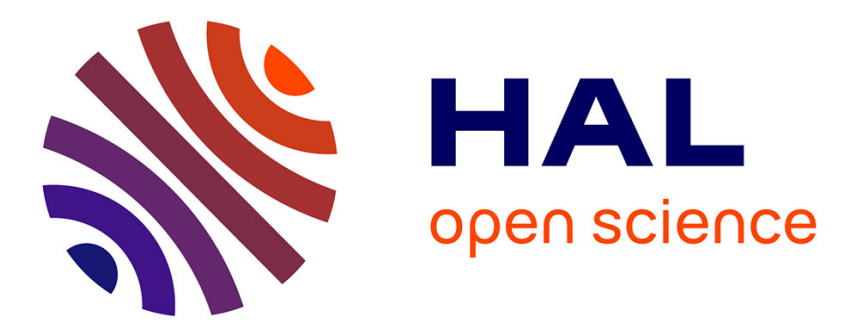

\title{
Spectral Unmixing for the Classification of Hyperspectral Images at a Finer Spatial Resolution
}

Alberto Villa, Jocelyn Chanussot, Jon Atli Benediktsson, Christian Jutten

\section{To cite this version:}

Alberto Villa, Jocelyn Chanussot, Jon Atli Benediktsson, Christian Jutten. Spectral Unmixing for the Classification of Hyperspectral Images at a Finer Spatial Resolution. IEEE Journal of Selected Topics in Signal Processing, 2011, 5 (3), pp.521-533. 10.1109/JSTSP.2010.2096798 . hal-00578890

\section{HAL Id: hal-00578890 \\ https://hal.science/hal-00578890}

Submitted on 22 Mar 2011

HAL is a multi-disciplinary open access archive for the deposit and dissemination of scientific research documents, whether they are published or not. The documents may come from teaching and research institutions in France or abroad, or from public or private research centers.
L'archive ouverte pluridisciplinaire HAL, est destinée au dépôt et à la diffusion de documents scientifiques de niveau recherche, publiés ou non, émanant des établissements d'enseignement et de recherche français ou étrangers, des laboratoires publics ou privés. 


\title{
Spectral Unmixing for the Classification of Hyperspectral Images at a Finer Spatial Resolution
}

\author{
A. Villa, Student Member, IEEE, J. Chanussot, Senior Member, IEEE, J. A. Benediktsson, Fellow, IEEE, \\ C. Jutten, Fellow, IEEE,
}

\begin{abstract}
The problem of classification of hyperspectral images containing mixed pixels is addressed. Hyperspectral imaging is a continuously growing area of remote sensing applications. The wide spectral range of such imagery, providing a very high spectral resolution, allows to detect and classify surfaces and chemical elements of the observed image. The main problem of hyperspectral data is the (relatively) low spatial resolution, which can vary from a few to tens of meters. Many factors make the spatial resolution one of the most expensive and hardest to improve in imaging systems. For classification, the major problem caused by low spatial resolution are the mixed pixels, i.e., parts of the image where more than one land cover map lie in the same pixel. In this work we propose a method to address the problem of mixed pixels and to obtain a finer spatial resolution of the land cover classification maps. The method exploits the advantages of both soft classification techniques and spectral unmixing algorithms, in order to determine the fractional abundances of the classes at a sub-pixel scale. Spatial regularization by Simulated Annealing is finally performed to spatially locate the obtained classes. Experiments carried out on synthetic real data sets show excellent results both from a qualitative and quantitative point of view.
\end{abstract}

Index Terms-Spatial resolution improvement, Hyperspectral data, Source separation, Simulated annealing, Spatial regularization

\section{INTRODUCTION}

Land cover classification of remote sensing data is an important application of image analysis. It is used in many practical applications, such as precision agriculture, monitoring and management of natural disasters, issues related to security and defense. The continuously growing availability of hyperspectral imagery, which records hundreds of images corresponding to different wavelength channels, has opened new possibilities in the field of image analysis and classification [1]. Hyperspectral sensors are characterized by a very high spectral resolution and a spatial resolution which can vary from few to tens of meters.

One of the major issues of hyperspectral images is that as the spectral resolution increases, the spatial resolution usually gets

A. Villa is with the GIPSA-lab, Signal \& Image Dept., Grenoble Institute of Technology - INP, France, and with the Faculty of Electrical and Computer Engineering, University of Iceland, Reykjavik, Iceland. (E-mail: alberto.villa@hyperinet.eu).

J. Chanussot is with the GIPSA-lab, Signal \& Image Dept., Grenoble Institute of Technology - INP, France. (E-mail: jocelyn.chanussot@gipsalab.grenoble-inp.fr).

J. A. Benediktsson is with the Faculty of Electrical and Computer Engineering, University of Iceland, Reykjavik, Iceland. (E-mail: benedikt@hi.is).

C. Jutten is with the GIPSA-lab, Signal \& Image Dept., Grenoble Institute of Technology - INP, France. (E-mail: christian.jutten@gipsa-lab.grenobleinp.fr). worse, and low spatial resolution is quite common, especially in case of high altitude sensors or instruments which cover wide areas [2]. These sensor limitations can affect the performances of algorithms used to process hyperspectral data. In classification, the relatively low spatial resolution can lead to the challenging problem of mixed pixels (Fig. 1), i.e., pixels containing more than one land cover type [3]. Also in case of high spatial resolution, a hyperspectral image is often a combination of pure and mixed pixels.

A number of so-called full pixel techniques, based on the assumption that each pixel corresponds to the spectral signature of one predominant land cover type, have been proposed during the last decades for the classification of hyperspectral images. [1], [4]. These techniques are not suitable for the analysis of mixed pixels and will inevitably lead to a high error rate when used for scenarios with a high number of sites with mixtures of land cover classes. The issue of mixed pixels has been considered in several works. A widely investigated approach is the use of soft classification techniques [5]. These classifiers do not assign a pixel to only one class, but they produce a set of images (one per class) that express for each pixel the degree of membership in the class in question [5]. However, the membership degree does not necessarily reflect the fractional abundance of a class within a mixed pixel, and the probability of a pixel to belong to one class does not necessarily correspond to the fractional part of the pixel covered by the considered class.

Linear spectral mixture analysis (SMA) [6] is a soft classification technique explicitely designed to address this problem. Following the spectral mixing model, the spectral signature of a mixed pixel is assumed to be the weighted sum of some constituent spectra, also called endmembers. Spectral unmixing is the procedure by which the measured spectrum of a pixel is decomposed into a collection of endmembers, and a set of corresponding fractions, or abundances, that indicate the proportion of each endmember within the pixel. A number of techniques, exploiting both statistical and geometrical properties of the data, was proposed over the last few years [7], [8]. These techniques can partially overcome the weakness of full pixel methods when analysing mixed pixels. However, when used to obtain crisp classification maps, the endmembers selection and the abundances determination are negatively affected by spectral variability [9], and common hard classification methods are more suitable in such a case [10].

Hard classification and spectral unmixing techniques can 


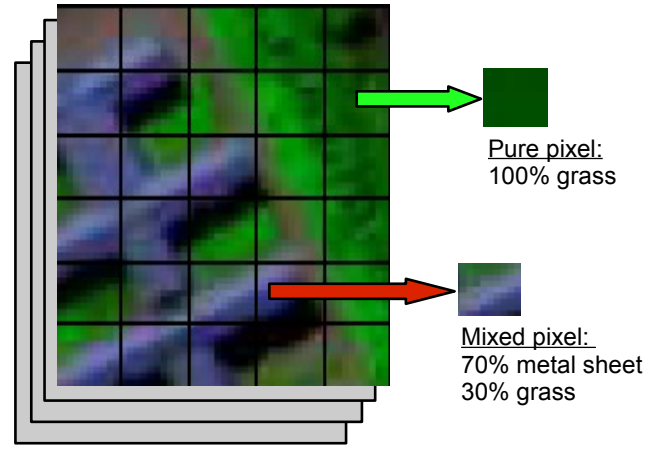

Fig. 1. Illustration of the problem of mixed pixels: When the spatial resolution is not fine enough, several land cover classes lie in the same pixel. In this case, a hard classification process cannot give an accurate information about the pixel coverage, leading inevitably to a loss of information

be seen as complementary methods, since the former are more suitable for the classification of pixels dominated by a single land cover class, while the latter are devoted to the mixed pixels analysis. Since a hyperspectral image usually contains many areas with pure pixels and some others with mixed, the combination of these two techniques can be seen as an interesting approach for the analysis of hyperspectral data. In spite of the fact that full pixel techniques and spectral unmixing methods could be combined in order to obtain improved classification maps in terms of accuracy, only few attempts have been made so far in order to jointly use these techniques, especially in [10], [11]. Plaza et al. [11] proposed an unsupervised approach for the classification of mixed pixels in hyperspectral imagery, based on the generalization of the concept of extended morphological profiles to case of multi bands images. The comparison of the method with commonly used techniques when applied to images with a low number of land cover classes or in case of high classes spectral separability showed interesting results. However, due to its unsupervised nature, the algorithm is expected to encounter difficulties when applied to more challenging data sets. An extension of the SVM classification technique to address the problem of mixed pixels was recently proposed in [10]. This extension provides interesting results when applied to synthetic data.

In this paper, a new supervised technique, which takes advantage of both probabilistic classification and spectral unmixing mapping techniques, is proposed in order to handle the issue of mixed pixels. The concept of sub-pixel mixing is also considered, in the attempt of obtaining land cover maps with an improved spatial resolution. The idea of subpixel mapping was first presented by Atkinson in [12]. He proposed to use the output of a soft classification technique in order to obtain a super-resolution mapping, trying to maximize the spatial correlation of the land cover classes to determine subpixels spatial locations. Since then, a number of techniques focused on better estimating sub-pixel fractional abundances determination and obtaining land cover maps with higher spatial resolution have been proposed [13]. In this paper, we propose the use of Simulated Annealing (SA) for this purpose, due to its simplicity and ease of use. This method has shown good results in a number of optimization and real problems, and its wide range of parameters grants a high flexibility with respect to the analysed problem. In multi-hyperspectral remote sensing, it has successfully been used for classification [14], [15], and abundances estimation [16].

The method proposed in this paper is in three steps. In a first step, a coarse classification is performed, based on the probabilistic output of an SVM. Every pixel can be assigned to a class, if the probability value obtained in the classification process is greater than a chosen threshold, or be unclassified. Pixels with a low probabilistic output are either mixed pixels or pixels hard to classify due to spectral variability, and their classification is addressed in a second step. In the second step, spectral unmixing is performed on the unclassified pixels by considering the preliminary results of the coarse classification step and by applying a Fully Constrained Least Squares (FCLS) method to every unlabeled pixel, in order to obtain the abundances fractions of each land cover type. Finally, in a third step, spatial regularization by SA is performed to obtain the resolution improvement. Experiments are carried out on synthetic and real hyperspectral data sets. The results are excellent both numerically and visually and show that the proposed method clearly outperforms traditional hard classification methods when the data contain mixed pixels.

The remainder of the paper is organized as follows. Section II presents in greater details the proposed approach. Section III shows the experiment on a synthetic data set, while Section IV illustrates the experimental results on real hyperspectral data. Section V finally draws the conclusions.

\section{Methodology}

The flow chart scheme of the proposed approach is presented in Fig. 2. The hyperspectral data are used as input for the hard classification method, in order to obtain a preliminary classification of all the pixels considered as "pure". The results of this step are the input (along with the original hyperspectral image) for the spectral unmixing, so that an appropriate set of endmembers can be found and the negative impact of spectral variabiality on the classification map minimized. In the last step, the results obtained are processed with a Simulated Annealing algorithm. Based on the assumption of spatial correlation of the land cover classes, SA is used to optimize a function where spatial proximity of pixels belonging to the same land cover class are preferred to the opposite case.

\section{A. Pixel-wise classification}

The first step of the proposed method consists in performing a pixelwise classification of the hyperspectral image, in order to obtain, for every pixel, a probability value for it to belong to one of the land cover classes. The pixels with a probability higher than a chosen treshold are considered as pixels where a single class is represented, and thus assigned to the considered class. These pixels are going to provide a preliminary classification map, where only the pixels containing a predominant 


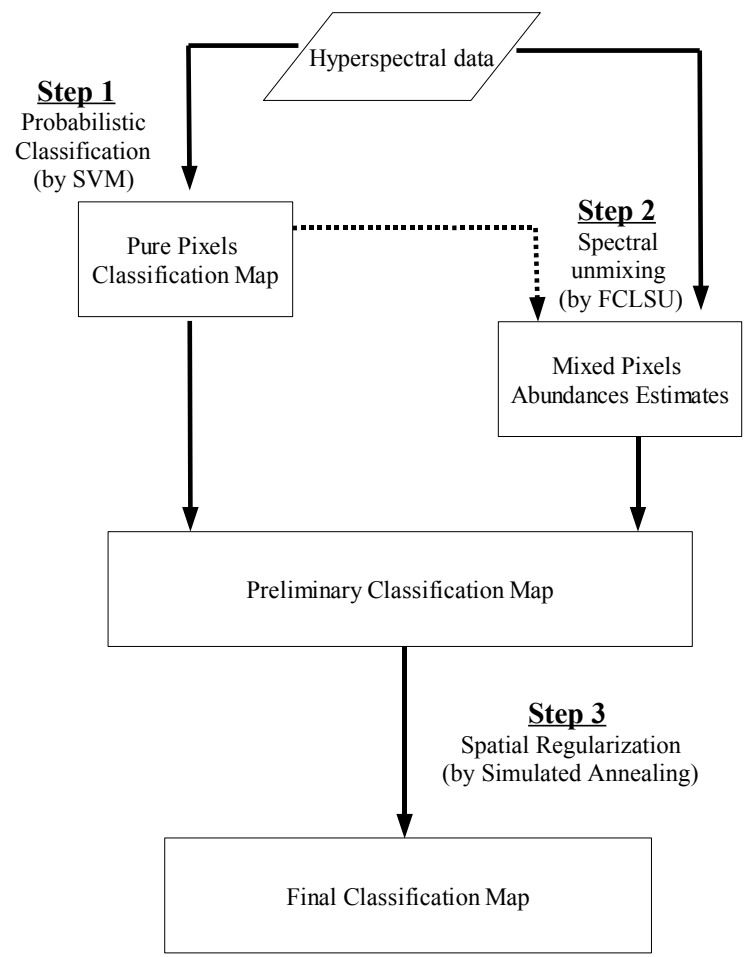

Fig. 2. Flow chart scheme of the proposed approach

land cover class are labeled. All the other pixels are not labeled, and their classification will be addressed in a second step.

As long as it can provide a probabilistic output, every classifier can be used for the data analysis. In this work, we propose to use a probabilistic Support Vector Machine (SVM) classifier [17], due to the good performances shown in the classification of hyperspectral data, also in case of limited training sets [18]-[20]. In the following, we give a short description of the principles on which SVM is based. Due to the lack of space, we refer the reader to [17] and [18] for further details on the theory of SVM and its application for hyperspectral images classification.

1) Probabilistic Support Vector Machine: The SVM is surely one of the most commonly used kernel learning algorithm. It performs robust non-linear classification of samples using the kernel trick [21]. The idea is to find a separating hyperplane in some feature space induced by the kernel function while all the computations are done in the original space [17]. Given a training set $\mathcal{S}=\left\{\left(\mathbf{x}^{1}, y_{1}\right), \ldots,\left(\mathbf{x}^{\ell}, y_{\ell}\right)\right\} \in$ $\mathbb{R}^{n} \times\{-1 ; 1\}$, the SVM computes a decision function $\mathrm{f}(\mathrm{x})$ such that $\operatorname{sign}(f(x))$ can be used to predict the label of any test sample $\mathrm{x}$. The decision function is found by solving the convex optimization problem:

$$
\begin{array}{ll}
\max _{\alpha} g(\alpha)= & \sum_{i=1}^{\ell} \alpha_{i}-\frac{1}{2} \sum_{i, j=1}^{\ell} \alpha_{i} \alpha_{j} y_{i} y_{j} \widetilde{k}\left(\mathbf{x}^{i}, \mathbf{x}^{j}\right) \\
\text { subject to } & 0 \leq \alpha_{i} \leq C \text { and } \sum_{i=1}^{\ell} \alpha_{i} y_{i}=0
\end{array}
$$

where $\alpha$ are the Lagrange coefficients, $\widetilde{k}\left(\mathbf{x}_{i}, \mathbf{x}_{j}\right)=$ $k\left(\mathbf{x}_{i}, \mathbf{x}_{j}\right)+\delta_{i j} / C, k$ the kernel function, $C$ a constant that is used to penalize the training errors, $\delta_{i j}$ a function such that $\delta=1$ if $i=j, \delta=0$ otherwise. To be an acceptable kernel, $k$ should be a positive semi-definite function [21].

In [22] Platt proposes approximating the posterior class probabilities $P(y=1 \mid x)$ by a sigmoid function:

$$
P(y=1 \mid x) \approx P_{A, B}(f)=\frac{1}{1+\exp (A \hat{f}+B)}
$$

where $\hat{f}$ is an estimation of the decision function $\mathrm{f}(\mathrm{x})$ computed by the SVM, $A$ and $B$ two parameters that need to be optimized. The best parameter setting $\left(A^{\star}, B^{\star}\right)$ is determined by solving the following regularized maximum likelihood problem (with $N_{+}$of the $y_{i}$ 's positive, and $N_{-}$negative):

$$
\min _{z=A, B} F(z)=\sum_{i=1}^{l}\left(t_{i} \log \left(p_{i}\right)+\left(1-t_{i}\right)\left(1-\log \left(p_{i}\right)\right)\right.
$$

where

$$
\begin{gathered}
p_{i}=P_{A, B}\left(f_{i}\right), \quad t_{i}=\left\{\begin{array}{lll}
\frac{N_{+}+1}{N_{+}+2} & \text { if } & y_{i}=+1 \\
\frac{1}{N_{-}+2} & \text { if } & y_{i}=-1
\end{array},\right. \\
i=1, \ldots, l
\end{gathered}
$$

A detailed description of the method can be found in [22]. In this work, we have used an improved implementation of the above algorithm [23], which is included in the LIBSVM library [24].

In this first classification step, we consider two outputs:

1) A complete probability map, containing the probability estimates for each pixel to belong to the assigned class.

2) A coarse classification map of the pixels considered as 'not mixed', containing class labels for the samples with a probability belonging to the class higher than a chosen treshold.

It is not a straightforward task to choose the treshold to determine if a pixel should be considered as pure or mixed. When labelling the pure pixels, we are interested in correctly classifying most of the pure pixels, because of two already mentioned reasons: i) When dealing with pure pixels, the full pixel methods work better than spectral unmixing, ii) this preliminary classification will be the input of the second step, and a large number of correctly classified pure pixel helps to provide suitable endmember candidates for the mixed pixels. For this same reason, the misclassification of pure pixels could lead to critical issues and cause a large error in the spectral unmixing step, thus a tradeoff is observed. The experiments carried out to investigate this issue have shown that in general a high treshold (close to 80\%) allows to obtain a higher classification accuracy, since only the pixels which are reasonably sure to belong to a class are labeled. In Section III, it will be shown that the choice of this parameter is not crucial for the classification accuracy of the proposed method. 


\begin{tabular}{|c|c|c|}
\hline $\mathbf{7 5} \%$ & $\mathbf{8 0} \%$ & $\mathbf{9 0 \%}$ \\
$\mathbf{2 5 \%}$ & $\mathbf{2 0 \%}$ & $10 \%$ \\
\hline $10 \%$ & $60 \%$ & $\mathbf{5 5} \%$ \\
$85 \%$ & $40 \%$ & $40 \%$ \\
\hline $80 \%$ & $65 \%$ & $\mathbf{2 3} \%$ \\
$10 \%$ & $35 \%$ & $77 \%$ \\
\hline
\end{tabular}

(a)

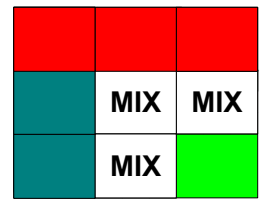

(b)

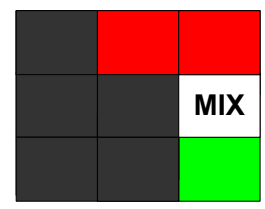

(c)

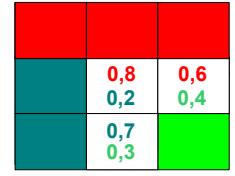

(d)

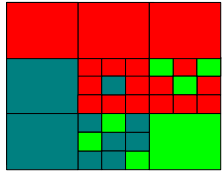

(e)

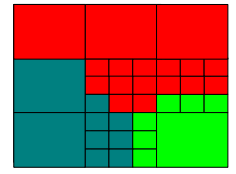

(f)

Fig. 3. Basic steps of the proposed method: (a) A probabilistic classification map is computed for each class. (b) The pixels with highest probability greater than a chosen treshold are considered as pure and classified (in the figure, we set the treshold to 70\%). The other pixels are considered as mixed (MIX in the figure). (c) For each mixed pixel, a set of possible endmembers is selected, considering the results of the preliminary classification. The other pixels, pure or mixed, are just ignored. (d) Spectral unmixing provides information about the abundance fraction of a class within each pixel. (e) Pixels are split into $n$ sub-pixels, according to the desired zoom factor, assigned to an endmember and randomly positioned within the pixel. The number of sub-pixels assigned to each class reflects the fractional value estimated in the previous step. (f) Simulated annealing performs random permutations of the sub-pixels position until minimum cost is reached.

\section{B. Spectral Unmixing}

After obtaining a coarse classification map, where some pixels considered as "pure" (due to the high probability to belong to the assigned class) were classified, the labelling of the other pixels is addressed in the second step.

Spectral mixture analysis (SMA) techniques have overcome some of the weaknesses of full pixel approaches by using linear statistical modeling and signal processing techniques [6], [8]. They are inherently either nonlinear techniques or linear techniques. Nonlinear mixed pixel analysis estimate multiple scattering effects that may arise when the different materials form intimate association at microscopic level [6]. Although they can be useful for some types of analysis, in the majority of applications a linear mixing model can be considered without significant loss of information [6]. The key task in linear SMA is to find an appropriate set of pure spectral constituents -called "endmembers" in hyperspectral analysis terminology-, which are then used to estimate the fractional abundances of each mixed pixel from its spectrum and the endmember spectra by using a linear mixture model. In the Linear Mixture Model (LMM), the spectrum of a mixed pixel is represented as a linear combination of component spectra (endmembers). The weight of each endmember spectrum (abundance) is proportional to the fraction of the pixel area covered by the endmember. If there are $M$ spectral bands, the spectrum of the pixel and the spectra of the endmembers can be represented by $M$-dimensional vectors. Therefore, the general equation for LMM is described as a linear regression form

$$
\mathbf{z}=\sum_{\mathbf{i}=\mathbf{1}}^{\mathbf{L}} \mathbf{a}_{\mathbf{i}} \mathbf{s}_{\mathbf{i}}+\mathbf{e}=\mathbf{A} \mathbf{s}+\mathbf{e}
$$

where $\mathbf{z}$ is an $M \times 1$ column pixel vector which describes the spectrum of the mixed pixel, $\mathbf{s}=\left[\mathbf{s}_{\mathbf{1}} \mathbf{s}_{\mathbf{2}} \ldots \mathbf{s}_{\mathbf{L}}\right]$ is an $M \times L$ endmember matrix of material signature, $\mathbf{s}_{\mathbf{i}}(i=1,2, \ldots, L)$ are the $M$-dimensional spectra of the endmembers, $\mathbf{a}$ is an $L$ $\times 1$ column vector and is composed of abundance coefficients $\mathbf{a}_{\mathbf{i}}(i=1,2, \ldots, L)$, e is an $M$-dimensional error vector accounting for lack-fit and noise effects, and $L$ is the number of the endmembers. Due to physical reasons, (4) has to respect the following constraints of non-negativity (abundance fractions within a pixel cannot be negative) and sum to one (the sum of all the abundances fraction within a pixel must have 1 as a result):

$$
\begin{aligned}
a_{i} & \geq 0 \\
\sum_{i=1}^{L} a_{i} & =1 .
\end{aligned}
$$

In recent years, several algorithms have been developed for automatic or semi-automatic extraction of spectral endmembers directly from the image data and to determine their fractional abundances within each pixel [7]. Assuming that ground truth is available, we do not need to determine the endmembers composing the data, but simply the abundance of each land cover type within the pixels. In this case, a major issue is how to handle the spectral variability which affects the data. As shown in [25], soft classification of hyperspectral images covering wide areas is negatively related to the intraclass spectral-variability, and the assumption that a single endmember could extensively represent a class is generally far from reality. The choice of appropriate endmembers is very important in order to correctly estimate the fractional abundances. If the endmembers do not represent the land cover classes well, the estimates of the sub-pixel coverage can be highly biased and lead to misclassification errors.

In order to overcome this problem, we propose an adaptive approach to select the best endmember candidates for each pixel. This approach is based on two main assumptions:

1) The spatial correlation of the classes, i.e., for each pixel, it is probable that the best endmember candidates lie in the spatial proximity of the considered pixel.

2) The probabilistic output provided by the SVM, i.e., if a candidate is not spatially close to the selected pixel, but the probabilistic value of the class to which it belongs is high, it is presumably a good candidate.

For each mixed pixel which has to be classified, we consider a set of 10 different spectra, that represent the endmember candidates. These candidates are chosen from the labeled samples of the training data and the results of the preliminary classification of step one, considered as a set of pure pixels correctly classified. If one of the land cover classes has a high probabilistic output (we consider a probabilistic output as high if its difference from the treshold chosen at step 1 is smaller than 5\%), at least five spectra of this class are 


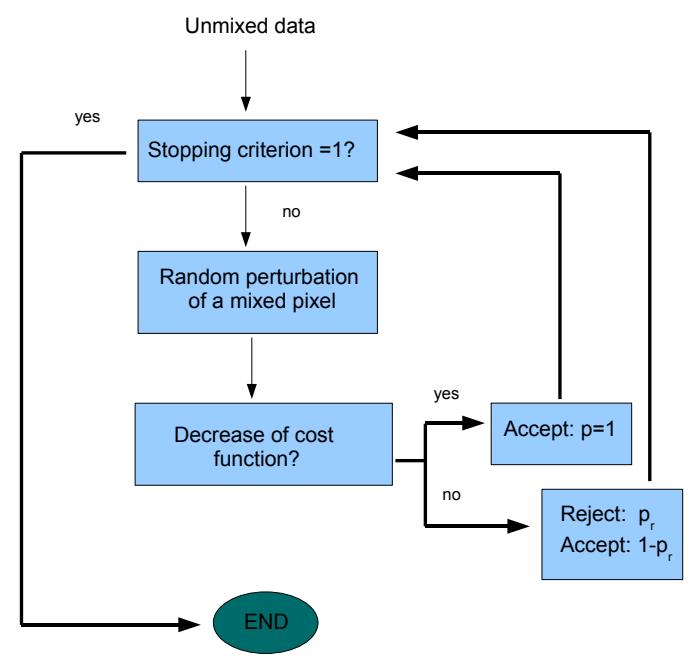

Fig. 4. Flow chart scheme of the proposed spatial regularization approach based on Simulated Annealing.

considered, otherwise all the 10 candidates are selected from the spectral signatures spatially closest to the considered pixel, after the coarse classification step. Once the spectral signatures representative of each class are extracted from the image, the abundance fraction of the elements within each pixel should be determined. Several algorithms have been developed for the linear mixing model according to the required constraints of abundances fractions. The fully constrained least squared unmixing algorithm is a widely adopted practical solution to avoid the appearance of theoretical problems, such as negative fractional abundances or abundances that sum up to more than one. Due to its computational efficiency, we have chosen this algorithm, which satisfies both abundance constraints and is optimal in terms of least squares error [26]. After applying FCLS, we obtain the fractional abundances of each endmember. Due to the fact that in many cases several endmembers represent the same class, by summing the fractional abundances of all the endmembers belonging to the same land cover class, we obtain the cover percentage of a class within a mixed pixel. It should be noted that not all the pixels to be classified in this second step are mixed, but there are many 'pure' pixels not labeled because of the low probability output provided by the SVM. However, the proposed method allows to label them as 'pure' pixels also in this second step.

\section{Improving Spatial Resolution}

Spectral unmixing is useful to describe the scene at a subpixel level, but can only provide information about proportions of the endmembers within each pixel. Since the spatial location remains unknown, spectral unmixing does not perform any resolution enhancement. In this paper, we propose a sub-pixel mapping technique, which takes advantage of the information given by the spectral mixing analysis and uses it to enhance the spatial resolution of thematic maps. Our proposed approach is as follows: In a first step, each pixel is divided in a fixed number of sub-pixels, according to the desired resolution enhancement. Every sub-pixel is assigned to an endmember, in conformity with its fractional abundance within the pixel. For example, if we want to have a zoom factor of $\mathrm{N}$, we have to divide each pixel into $\mathrm{N} \times \mathrm{N}$ sub-pixels. For each pixel, the number of subpixels $n$ to assign to the class $i$ is computed according to the equation:

$$
n_{i}=\operatorname{round}\left(\frac{\operatorname{abd}_{i}}{1 / N}\right)
$$

where $\operatorname{abd}_{i}$ is the fractional abundance of the class $i$ within the considered pixel estimated with the FCLS and round(x) returns the value of the closest integer to $\mathrm{x}$.

A Simulated Annealing (SA) mapping function is then used, to create random permutation of these sub-pixels, in order to minimize a chosen cost function. Relying on the spatial correlation tendency of landcovers, we assume that each endmember within a pixel should be spatially close to the same endmembers in the surrounding pixels. Therefore, the cost function $C$ to be minimized is chosen as the perimeter of the areas belonging to the same class:

$$
C=\sum_{i=1}^{I} \sum_{j=1}^{N_{i}} P_{j},
$$

where $I$ is the number of the classes, $N_{i}$ is the number of connected components of the class $i$, and $P_{j}$ is the perimeter of the connected component $j$, computed according to the 8connected border pixels model [27].

SA is a well established stochastic technique originally developed to model the natural process of crystalization [28]. This process is based on an analogy from thermodynamics where a system is slowly cooled in order to reach its lowest energy state. More recently, SA has been proposed to solve global optimization problems [29], and it has been used in various fields. The basic idea of the method is that, in order to avoid to be trapped in local minima, uphill movements, i.e., points corresponding to worse values of the objective function could, sometimes, be accepted for the following iteration. As with a greedy search, it accepts all the changes that improve the solution. Changes degrading the solution can be accepted, but with a probability that is inversely proportional to the size of the degradation (small degradations are accepted with a higher probability). This probability also decreases as the search continues, or as the system cools down, allowing eventual convergence to the optimal solution.

An example of how SA spatial regularization works can be seen by looking at Fig.3, where 3 (e) represents the initial sub-pixel distribution and 3 (f) the optimal one. First, a mixed pixed is selected, according to the information provided by the spectral unmixing step. Then, a random permutation of the subpixels within the chosen pixel is performed by SA. If this permutation leads to a decrease of the cost function (which is in our case the perimeter of connected components, i.e., components belonging to the same class), the change is accepted. Otherwise, as decribed above, the change will be probably rejected. The algorithm stops when minimum cost is reached, that is when a previously fixed number of 
TABLE I

DESCRIPTION OF THE PURENESS OF EACH PIXEL. ABD REPRESENTS THE maximum VALUE OF ABUNDANCE WITHIN A PIXEL

\begin{tabular}{|c|c|}
\hline Pureness & Number of Pixels \\
\hline abd $>95 \%$ & 7105 \\
$95 \%>$ abd $>85 \%$ & 828 \\
$85 \%>$ abd $>75 \%$ & 484 \\
$75 \%>$ abd $>65 \%$ & 576 \\
$65 \%>$ abd $>55 \%$ & 384 \\
abd $<55 \%$ & 323 \\
\hline
\end{tabular}

consecutively rejected changes is reached (in our case, we set the number of consecutive operations to 100.000 , since it is large enough to avoid sub-optimal solutions and Matlab takes only a few seconds to perfom this computation).

\section{EXPERIMENTS ON SIMULATED DATA}

The first experiment was carried out on a synthetic data set. The advantage of using a synthetic data set is the perfect knowledge that we have about the analysed image. This experiment has two main aims: the first one is to verify the assumption that the pixels classified by the SVM with a high probability value effectively correspond to 'pure' pixels. The second one is to validate the proposed method with a data set known in details, in order to evaluate the performances under different conditions of mixtures. The creation of a synthetic data set for classification purposes is not a trivial task, especially in this case where the spatial information is very important to fully exploit the potentiality of the proposed method. In order to build a data set as realistic as possible, we have considered a thematic map of an AVIRIS image, taken over the area of San Diego, with 9 land cover classes. We have created a hyperspectral data cube by substituting every class with a spectrum taken from the USGS spectral library, available on-line [30]. The classes were chosen mainly from the vegetation library, in order to make more difficult the discrmination. The chosen classe were: shadow, asphalt, green grass, dry grass, maple, pine lodgepole, pine white, pinon and rub. The original image created was $400 \times 400$ pixels. Gaussian noise was added in order to reach a SNR of $30 \mathrm{~dB}$. To have the possibility to analyse data sets where the ground truth cover is known in details, and to evaluate the obtained results from a quantitative point of view, we decided to use the original ground truth data only to compare the obtained results, and to decrease the spatial resolution of the image by applying an $4 \times 4$ low pass filter, so that we obtained an image of the same area with a resolution degraded of a factor 4 . This way, we have the possibility to test the proposed method on a data set know in details, where there are pure, close to pure, and mixed pixels. The complete description of the data set is given in Table I. In order to compare the proposed approach with a common hard classification method, the same data were also classified with an SVM wih Gaussian Kernel, One vs One multiclass strategy and 10 fold cross-validation. Among the several multi-class strategies available for the SVM, we have chosen the One vs One because of its good performances in terms of robustness and computational burden [31]. The comparison of the low resolution map obtained with SVM to the high resolution ground truth was not possible due to different number of pixels of the two images. However, we know that every pixel of the low resolution image corresponds to $n \times n$ pixels of the high resolution image. By comparing a pixel of the low resolution classification map with the $n \times n$ corresponding in the high resolution ground truth map, we can compute per-pixel classification accuracy. By doing this, we have to keep in mind that in case of a mixed pixel the hard classification method will inevitably lead to an error, because it will assign the corresponding high resolution $n \times n$ pixels to just one class, considered as predominant within the mixed pixel. However, this is exactly the issue that the proposed method is expected to address.

The performances of the two methods were compared in terms of overall accuracy (OA), that is the number of correctly classified test samples with respect to the total number of test samples, average accuracy (AA), which represents the average of the classification accuracies for the individual classes, and the Kappa coefficient of agreement $(\kappa)$, that is a parameter that estimates the correct percentage classification without the amount that could be expected due to chance alone [32]. In addition to this, we have computed the number of mixed pixels correctly classified, in order to show the performances of the two methods when dealing with mixtures of classes, and the number of pixels correctly labeled after the spectral unmixing step but uncorrectly positioned after Simulated Annealing.

For each class, $2 \%$ of the labeled samples were selected for training the algorithm. The treshold between pure and mixed pixels has been set to 0.7 . The results of the experiment are shown in Table II. The first three columns represent the results obtained on the entire data set in terms of OA, $\kappa$ and AA, and show that the proposed method provide an improvement in the overall accuracy classification. The second part of the Table II show a comparison of the performance of the two methods over groups of pixels with different degrees of purity, varying from pure pixels (where the predominant class has an abundance larger than 95\%) to highly mixed pixels (where the predominant class has an abundance smaller than 55\%). It can be noticed that while in case of pure and close to pure pixels the results of the two methods are quite similar, the proposed approach provided a dramatic increase of classification accuracy for mixed and highly mixed pixels, where a traditional classifier completely fails while the proposed method improves the accuracy of up to 35 percentage points. In order to evaluate the correctness of the assumption that pure pixels are classified with a high probability value, we have computed the mean probability of the maximum value of each group of pixels and the number of pixels of each group which are classified with a probability value higher than $70 \%$ (two leftmost columns of Table II). It can be easily noticed that the larger is the maximum value of abundance within the pixel, the higher is the probabilistic output provided by the SVM. The $80 \%$ of pure pixels were classified with a probability larger than 0.7 , while in case of mixed and highly mixed pixels this quantity 
TABLE II

RESULTS OF THE EXPERIMENT ON THE SYNTHETIC DATA SET. ABD REPRESENT THE maximum VALUE OF ABUNDANCE WITHIN A PIXEL. THE THIRD COLUMN DESCRIBES THE AVERAGE OF THE MAXIMUM PROBABILITY VALUE WITHIN A GROUP OF PIXELS WITH THE SAME PURITY DEGREE. THE LAST COLUMN REPRESENTS THE NUMBER OF PIXELS WITH A MAXIMUM PROBABILITY VALUE GREATER THAN 0.7, ACCORDING TO THEIR PURITY DEGREE.

\begin{tabular}{|c||c|c||c|c|}
\hline & SVM & Proposed Method & Average Probabilistic SVM Output & Max(prob) $>0.7$ \\
\hline OA & $88.21 \%$ & $90.84 \%$ & - & - \\
$\kappa$ & $87.34 \%$ & $89.52 \%$ & - & - \\
AA & $88.32 \%$ & $90.25 \%$ & - & - \\
\hline abd $>95 \%$ & $99.50 \%$ & $99.50 \%$ & 0.74 & 5317 \\
$95 \%>$ abd $>85 \%$ & $92.25 \%$ & $91.44 \%$ & 0.68 & 328 \\
$85 \%>$ abd $>75 \%$ & $79.57 \%$ & $77.57 \%$ & 0.54 & 27 \\
$75 \%>$ abd $>65 \%$ & $54.12 \%$ & $61.17 \%$ & 0.39 & 5 \\
$65 \%>$ abd $>55 \%$ & $34.82 \%$ & $50.33 \%$ & 0.30 & 0 \\
abd $<55 \%$ & $15.36 \%$ & $40.22 \%$ & 0.26 & 1 \\
\hline
\end{tabular}

drops to $0.4 \%$.

\section{EXPERIMENTS ON REAL DATA}

The experiments on real data were carried out considering three different data sets from two hyperspectral images. The first two data sets are from an Airborne Visible/Infrared Imaging Spectrometer (AVIRIS) image taken over NW Indiana's Indian Pine test site in June 1992. This image has been widely used in the remote sensing community for both classification and spectral unmixing purposes, and thus represents an interesting benchmark for the proposed method. According to [33], we considered for the first experiment a part of the scene, consisting of pixels [31-116] $\times$ [27-94] for a size of $86 \times 68$, which contains four labeled classes (the background pixels were not considered for classification purposes). We will refer to this data set as the "Subset scene". The second experiment was carried out on the whole AVIRIS data set. Sixteen land cover classes were considered. The original image is composed by $145 \times 145$ pixels. The calibrated data are available online ${ }^{1}$ with detailed ground-truth information.

Finally, the third study site is the region surrounding the central-volcano Hekla in Iceland, one of the most active volcanoes in the country. Since 1970, Hekla has erupted quite regularly every 10 years, in 1970, 1980-81, 1991 and in 2000. The volcano is located on the South-Western margin of the Eastern volcanic zone in South Iceland. Hekla's products are mainly andesitic and basaltic lavas and tephra. AVIRIS data that were collected on a cloud-free day, June 17 1991, were used for the classification. The AVIRIS sensor operates in the visible, near- and mid- infrared portions of the electromagnetic spectrum, its sensitivity range spanning wavelengths from 0.4 $\mu \mathrm{m}$ to $2.4 \mu \mathrm{m}$. As on the previous case, the sensor system has 224 data channels, utilizing four spectrometers, whereas each spectral band is approximately $10 \mathrm{~nm}$ in width. During the image acquisition, spectrometer 4 was not working properly. This particular spectrometer operates in the wavelength range from $1.84 \mu \mathrm{m}$ to $2.4 \mu \mathrm{m}$ (64 bands). These 64 bands were deleted from the imagery along with the first channels for all the other spectrometers, and the remaining 157 data channels

\footnotetext{
${ }^{1}$ http://dynamo.ecn.purdue.edu/ biehl/
}

were left. A subset of $180 \times 180$ pixels has been used for this experiment. In order to address the issue of the random choice of the training samples, for each data set we have repeated the experiment with ten different training sets.

As in the previous experiment, due to the difficulty to have a perfect knowledge of the fractional abundances of each land cover type, we decided to use the original ground truth data only to compare the obtained results, and to decrease the spatial resolution of the image by applying an $n \times n$ low pass filter, where $n$ varies according to the considered data set. This way, we know exactly the quantity of each class within a pixel, and we can use the low resolution image obtained after filtering as input for the proposed method. The information about the classes, the training and the test sets can be found in Table III.

\section{A. AVIRIS subset}

The first experiment was carried out on the AVIRIS subset image. The goal of this experiment is to illustrate the effectiveness of the method when used for the analysis of a simple hyperspectral data set. In this subimage, composed by $86 \times 68$ pixels, there are four classes with uneven number of labeled samples, namely, Corn-notill, Grass/Trees, Soybeans-no till, and Soybeans-min. The complete description of the training and test sets can be found in Table III. A $3 \times 3$ low-pass filter was applied to the original image, so that a new image with lower spatial resolution was obtained. The new image was composed by $28 \times 23$ pixels, and it was used as input for the proposed method. The low resolution image obtained after filtering and the ground truth can be seen in Fig. 5 (ab). Twenty pixels per class, considered as "pure" in the low resolution image, were randomly chosen and used for training the SVM classifier.

The result of the classification with the SVM is presented in Fig. 5 (c). As it can be seen from the classification map, the two main problems are represented by the mixed pixels, which make hard to distinguish the border between different land cover areas, and the high spectral variability, which results in a noisy classification map. The proposed method provides an overall improvement for both issues (classification map shown in Fig. 5 (f)). It can be seen at the top of the image that the border of the Corn-no till field (represented in light blue in the map) is estimated with improved accuracy 
TABLE III

INFORMATION ABOUT THE TRAINING AND THE TESTING SET OF THE THREE CONSIDERED DATA SETS. IT HAS TO BE NOTICED THAT THE TRAINING SET IS SELECTED FROM THE LOW RESOLUTION IMAGE USED AS INPUT OF THE METHOD, WHILE THE TEST SET IS SELECTED FROM THE HIGH RESOLUTION REFERENCE DATA USED FOR COMPARISON.

\begin{tabular}{|c||c|c|c|c|c|c|c|c|c|}
\hline \multicolumn{1}{|c||}{} & \multicolumn{2}{|c|}{ AVIRIS Indian Pine Subset } & \multicolumn{2}{c|}{ AVIRIS Indian Pine Complete } & \multicolumn{3}{c|}{ AVIRIS Hekla } \\
\hline No. & Name & Train & Test & Name & Train & Test & Name & Train & Test \\
\hline 1 & Corn-no till & 20 & 1434 & Alfa Alfa & 4 & 54 & Andesite lava 1970 & 24 & 672 \\
2 & Grass-Trees & 20 & 747 & Corn-no till & 44 & 1434 & And. lava 1980 I & 126 & 3350 \\
3 & Soybean-no till & 20 & 727 & Corn-min till & 25 & 834 & And. lava 1980 II & 523 & 11916 \\
4 & Soybean-min till & 20 & 1926 & Corn & 7 & 234 & And. lava 1991 I & 220 & 4709 \\
5 & - & - & - & Grass-Pasture & 15 & 497 & And. lava 1991 II & 279 & 6918 \\
6 & - & - & - & Grass-Trees & 23 & 747 & Lava tephra covered & 103 & 2310 \\
7 & - & - & - & Grass-Mowed & 4 & 26 & Rhyolite & 6 & 181 \\
8 & - & - & - & Hay-windrowed & 17 & 489 & Scoria & 51 & 1286 \\
9 & - & - & - & Oats & 4 & 20 & Firn-glacier ice & 42 & 1058 \\
10 & - & - & - & Soybean-no till & 32 & 968 & - & - & - \\
11 & - & - & - & Soybean-min till & 83 & 2468 & - & - & - \\
12 & - & - & - & Soybean-clean t & 19 & 614 & - & - & - \\
13 & - & - & - & Wheat & 5 & 212 & - & - & - \\
14 & - & - & - & Woods & 42 & 1294 & - & - & - \\
15 & - & - & - & Bldg-Trees-Drive & 12 & 380 & - & - & - \\
16 & - & - & - & Stone-Steel Tower & 4 & 95 & - & - & - \\
\hline
\end{tabular}

when compared to the traditional SVM classification, thus assessing the effectiveness of the proposed approach also to provide classification maps with a better spatial resolution. The problem of spectral variability is also solved, since the classification map is much less noisy and, therefore, a lower number of sparse pixels is observed.

The quantitative results obtained with the two methods are presented in Table IV, along with the results of the other data sets. The proposed method provides an improvement of the overall accuracy which is greater than $10 \%$. As it will be in the other experiments, the improvement of the classification accuracy of the mixed pixel with respect the SVM is larger than for the whole data set. This demonstrates that the proposed method is effective in improving the results of data sets with mixtures of land cover classes. To assess the effectiveness of SA to locate sub-pixels in the classification map, we have also computed the number of sub-pixels correctly classified after the spectral unmixing step, but uncorrectly located after the spatial regularization. In this case, we can see that the error due to bad positioning of sub-pixels is extremely low.

\section{B. AVIRIS complete}

The second experiment was carried out on the whole AVIRIS data set. Sixteen land cover classes were considered for classification. The original image is composed by $145 \times$ 145 pixels, and it was used as reference data. After applying a $2 \times 2$ low pass filter, an image composed by $72 \times 72$ pixels was obtained. The land cover ground truth can be seen in Fig. 6 (a). For training set, we have randomly selected, for each class, $15 \%$ of all the samples which were considered as "pure" in the low resolution image (that would correspond to about $10 \%$ of pixels of each class in the high resolution image). To have the possibility to compare the results of the proposed method with the available ground truth, we chose a zoom factor equal to 2 , lower than in the previous case. However, the higher number of classes and their spectral similarity make this data set more challenging than the first one.
Figure 6 (b) and (e) shows the classification maps obtained with a conventional SVM and the proposed method. Also in this case, an improvement can be clearly seen in the classification maps, resulting in a less noisy map and an improved detection of the borders of spatial structures (in this case, agricultural fields). To have a quantitative comparison of the results obtained with the two methods, the overall accuracy of pixels correctly classified has been compared. The mean overall accuracy obtained in the five experiments with the SVM is $72.31 \%$. As in the previous case, the low value of accuracy is due to two main factors, which are the impossibility of a common hard classification technique to distinguish different land cover classes at a sub-pixel level, and the difficulty to handle the high spectral variability. The proposed method obtained an average overall accuracy of $91.10 \%$, showing the capability of the proposed approach to better deal with the aforementioned two main issues. By comparing Figure 6 (d) and (e), it can be noticed the effectiveness of the proposed spatial regularization with Simulated Annealing.

\section{Hekla data set}

For the last experiment, we consider a subset of the Hekla data set, located in the top-left corner of the scene. This subset is composed by $180 \times 180$ pixels, and it contains nine classes of interest. Also in this case a $2 \times 2$ low pass filter was applied to the original image, leading to a low resolution image of $90 \times 90$ pixels. Due to the insufficient availability of ground truth to quantify the results provided by the proposed method, we have considered as ground truth the classification map obtained by a spectral-spatial method, proposed in [34], where the overall accuracy computed on the reference test set was close to $100 \%$. Thus, also if we have to keep in mind that the results are estimated by comparison with a classification map and not with a selected land cover ground truth map, this classification map seems to be a reliable source of knowledge about the land class coverage of the area. The original image, the classification map obtained in [34] and 


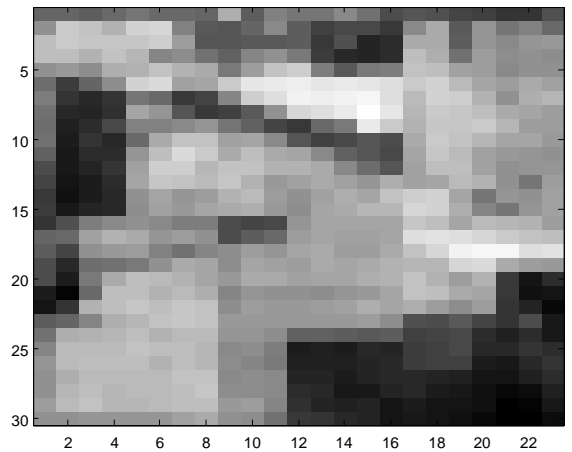

(a)

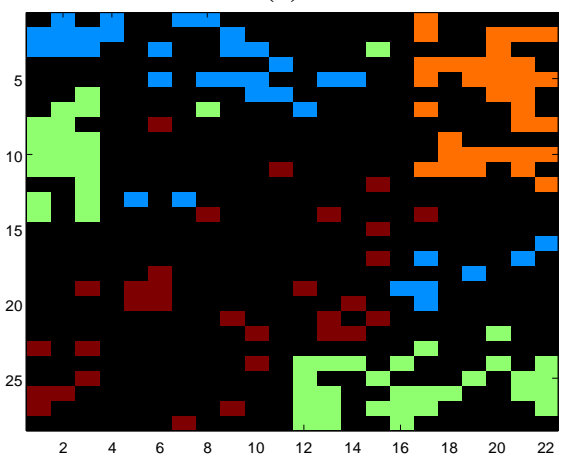

(d)

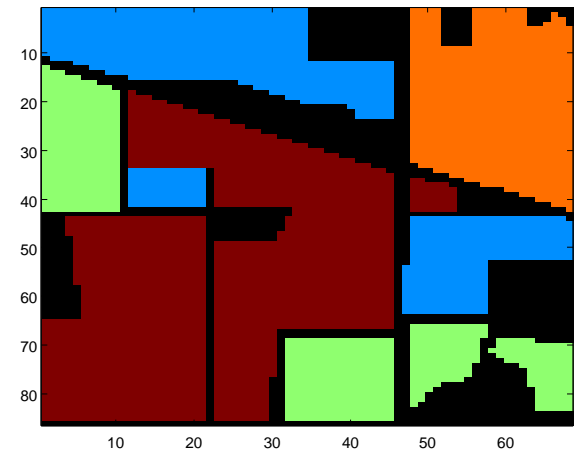

(b)

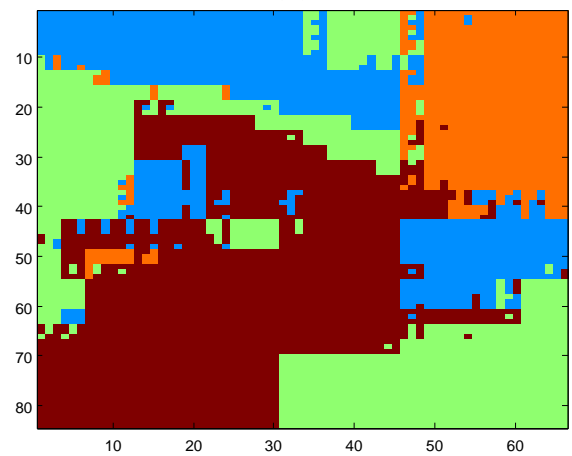

(e)

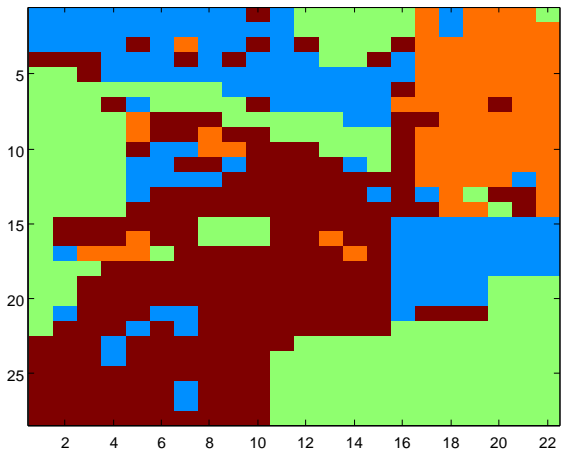

(c)

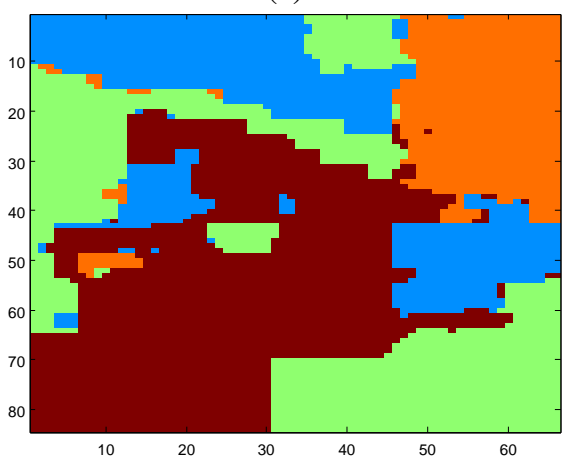

(f)

Fig. 5. AVIRIS subset data: (a) Low resolution image (band 30) obtained after applying a $3 \times 3$ filter to the high resolution image. (b) Ground truth of the high resolution image. Unknown pixels are represented in black. (c) Classification map obtained with traditional SVM. (d) Results of the preliminary classification. (e) Classification map obtained before applying the spatial regularization. (f) Final classification map obtained with the proposed method.

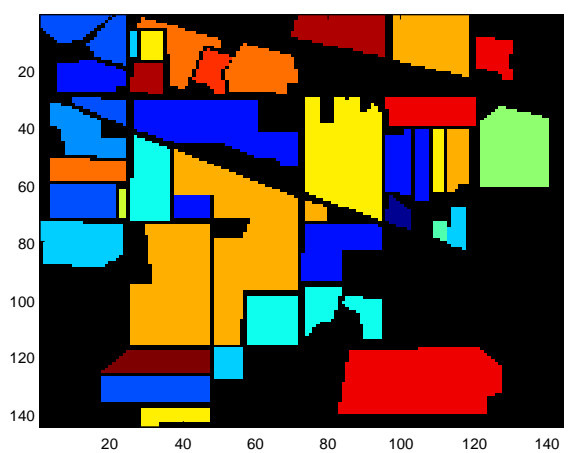

(a)

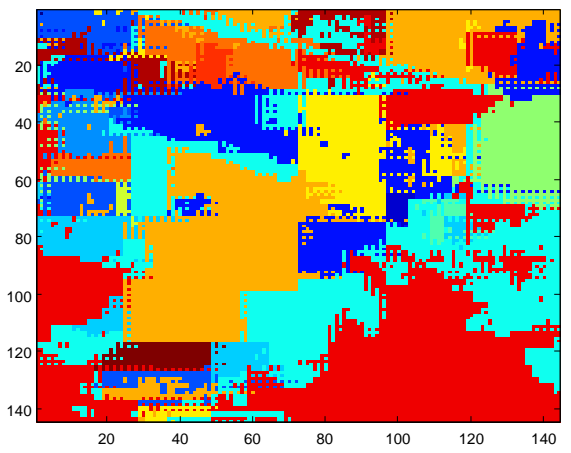

(d)

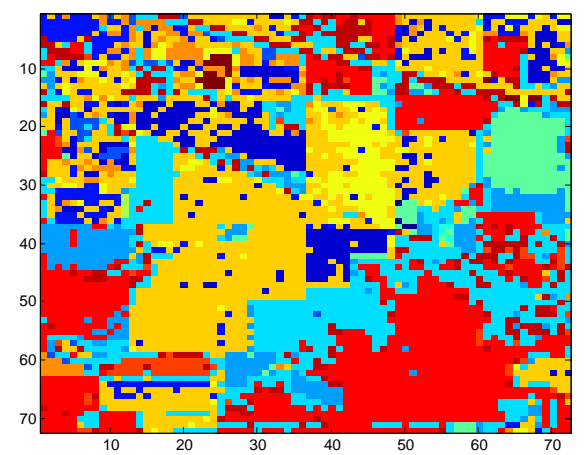

(b)

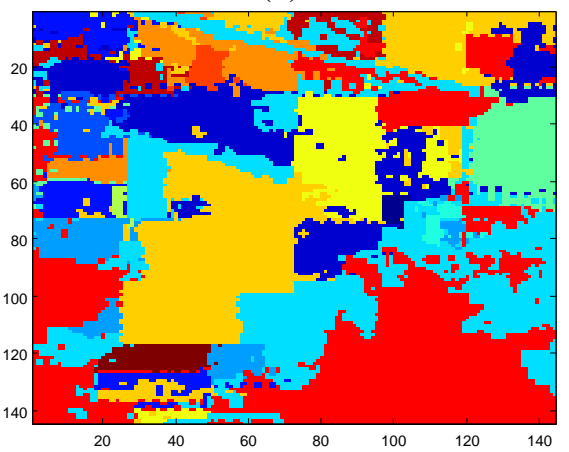

(e)

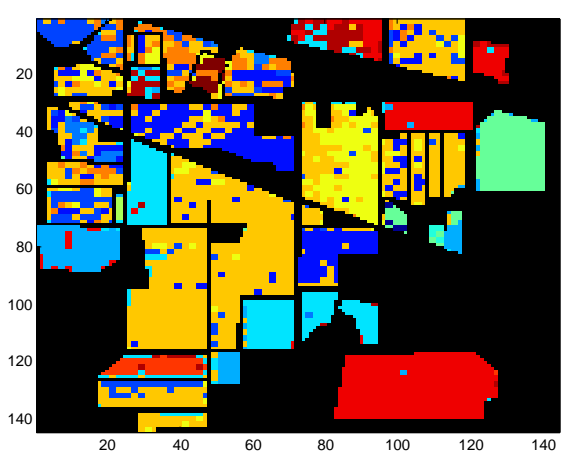

(c)

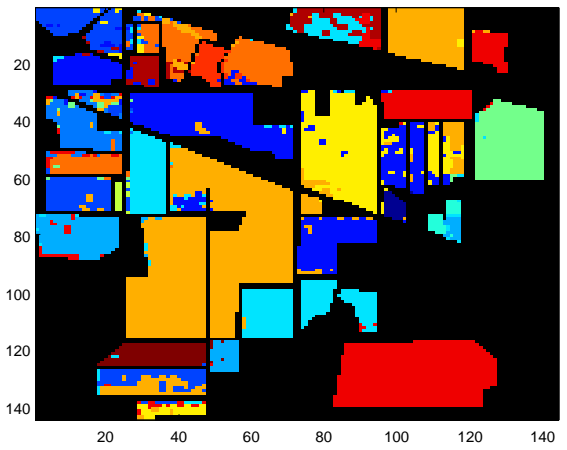

(f)

Fig. 6. (a) Ground truth data (b) Classification map obtained with an one versus one SVM (training set 1) (c) Classification of the ground truth pixels (d) Classification map obtained before applying the spatial regularization. (e) Final classification map obtained with the proposed approach. (f) Classification of the ground truth pixels. 


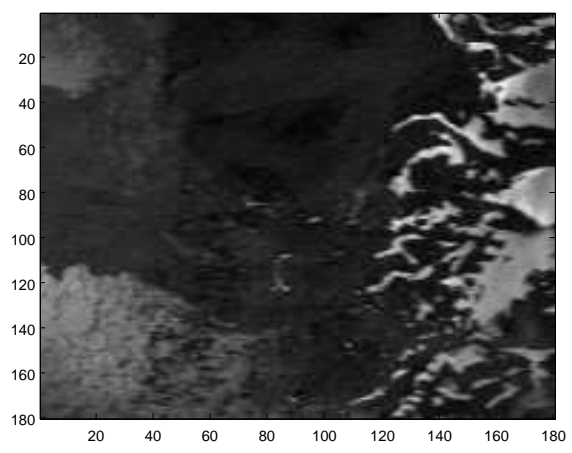

(a)

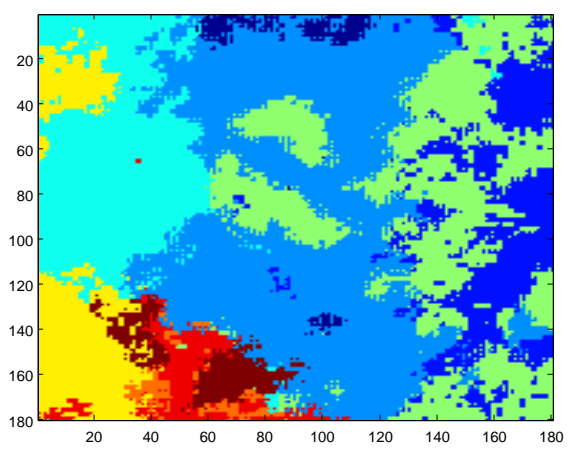

(d)

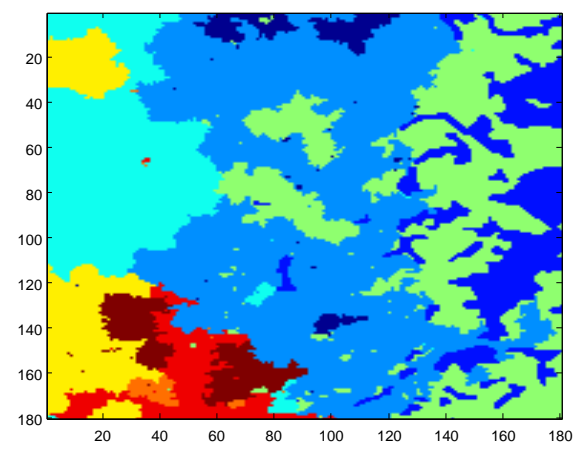

(b)

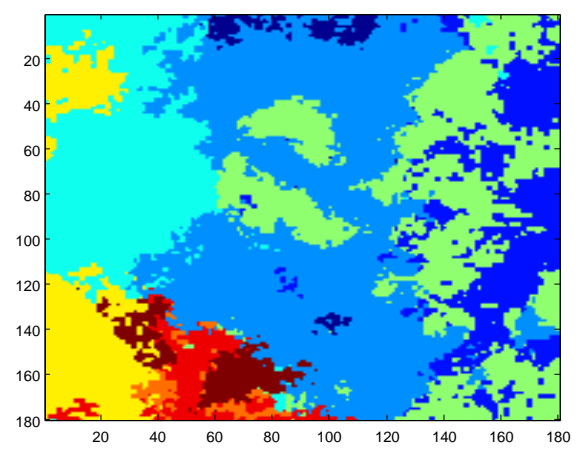

(e)

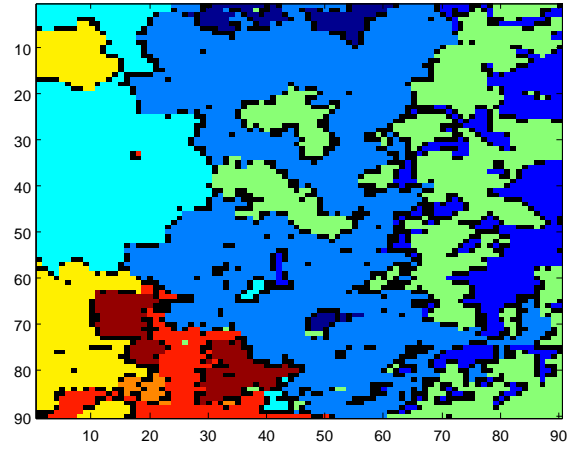

(c)

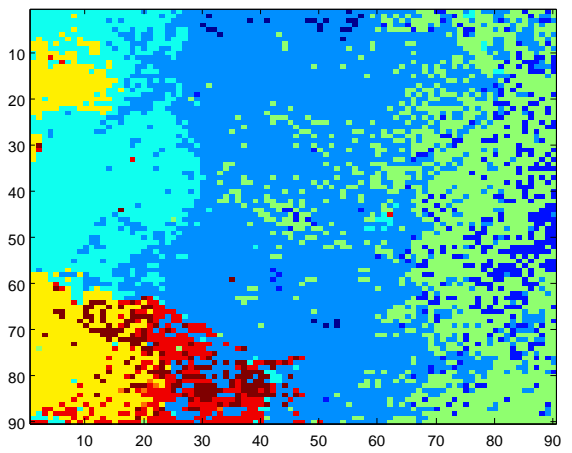

(f)

Fig. 7. (a) AVIRIS Hekla, band 80 (b) Classification map obtained in [34]; (c) Low resolution ground truth. In black are represented the mixed pixels (d) Classification map obtained with the proposed approach after spectral unmixing (training set 1, treshold 0.7) (e) Final classification map obtained with the proposed approach (f) Classification map obtained with SVM

TABLE IV

COMPARISON OF CLASSIFICATION ACCURACY OBTAINED WITH THE PROPOSED METHOD AND SVM IN THE THREE ANALYSED DATA SETS. "MIXED PIXELS" REFERS TO THE PERCENTAGE OF CORRECTLY CLASSIFIED MIXED PIXELS (I.E., PIXELS CONSIDERED AS PURE IN THE REFERENCE IMAGE BUT MIXED IN THE LOW RESOLUTION ONE). "SPATIAL ERROR" REFERS TO THE PERCENTAGE OF SUB-PIXELS CORRECTLY RETRIEVED AFTER SPECTRAL UNMIXING BUT UNCORRECTLY LOCATED AFTER SPATIAL REGULARIZATION.

\begin{tabular}{|c|c|c|c|c|c|c|}
\hline & \multicolumn{2}{|c|}{ AVIRIS Indian Pine subset } & \multicolumn{2}{c|}{ AVIRIS Indian Pine complete } & \multicolumn{2}{c|}{ AVIRIS Hekla } \\
\hline Approach & SVM & Proposed Method & SVM & Proposed Method & SVM & Proposed Method \\
\hline OA & $78.22 \pm 0.94 \%$ & $\mathbf{9 0 . 6 5} \pm \mathbf{2 . 4 1 \%}$ & $72.31 \pm 1.64 \%$ & $\mathbf{9 1 . 1 0} \pm \mathbf{1 . 4 2 \%}$ & $69.19 \pm 2.10 \%$ & $\mathbf{8 1 . 7 1} \pm \mathbf{2 . 3 4 \%}$ \\
$\kappa$ & $68.14 \pm 1.63 \%$ & $\mathbf{8 4 . 3 8} \pm \mathbf{3 . 7 6} \%$ & $67.53 \pm 1.78 \%$ & $\mathbf{8 8 . 8 4} \pm \mathbf{1 . 6 5 \%}$ & $63.96 \pm 1.91 \%$ & $\mathbf{7 6 . 2 3} \pm \mathbf{2 . 3 6 \%}$ \\
AA & $81.47 \pm 1.59 \%$ & $\mathbf{9 1 . 3 6} \pm \mathbf{1 . 2 1 \%}$ & $64.34 \pm 1.19 \%$ & $\mathbf{9 0 . 7 3} \pm \mathbf{1 . 7 3 \%}$ & $62.83 \pm 2.71 \%$ & $\mathbf{7 4 . 7 2} \pm \mathbf{3 . 5 0 \%}$ \\
Mixed Pixels & $73.85 \%$ & $88.13 \%$ & $50.21 \%$ & $72.77 \%$ & $48.10 \%$ & $67.65 \%$ \\
Spatial Error & - & $0.62 \%$ & - & $1.38 \%$ & - & $2.92 \%$ \\
\hline \hline Class 1 & $74.49 \%$ & $87.21 \%$ & $36.30 \%$ & $88.15 \%$ & $50.30 \%$ & $52.98 \%$ \\
Class 2 & $99.24 \%$ & $93.59 \%$ & $61.83 \%$ & $87.07 \%$ & $89.22 \%$ & $62.18 \%$ \\
Class 3 & $76.39 \%$ & $93.67 \%$ & $40.65 \%$ & $79.21 \%$ & $74.99 \%$ & $85.84 \%$ \\
Class 4 & $75.74 \%$ & $90.98 \%$ & $26.24 \%$ & $84.70 \%$ & $90.27 \%$ & $90.61 \%$ \\
Class 5 & - & - & $82.45 \%$ & $84.55 \%$ & $42.89 \%$ & $82.87 \%$ \\
Class 6 & - & - & $90.63 \%$ & $95.53 \%$ & $78.96 \%$ & $84.55 \%$ \\
Class 7 & - & - & $76.92 \%$ & $99.23 \%$ & $50.82 \%$ & $60.83 \%$ \\
Class 8 & - & - & $93.54 \%$ & $98.94 \%$ & $34.37 \%$ & $68.35 \%$ \\
Class 9 & - & - & $80.00 \%$ & $94.00 \%$ & $53.68 \%$ & $84.31 \%$ \\
Class 10 & - & - & $51.51 \%$ & $84.55 \%$ & - & - \\
Class 11 & - & - & $85.41 \%$ & $96.56 \%$ & - & - \\
Class 12 & - & - & $36.03 \%$ & $85.18 \%$ & - & - \\
Class 13 & - & - & $66.79 \%$ & $99.06 \%$ & - & - \\
Class 14 & - & - & $92.92 \%$ & $99.30 \%$ & - & - \\
Class 15 & - & - & $44.47 \%$ & $84.32 \%$ & - & - \\
Class 16 & - & - & $63.79 \%$ & $91.37 \%$ & - & - \\
\hline
\end{tabular}




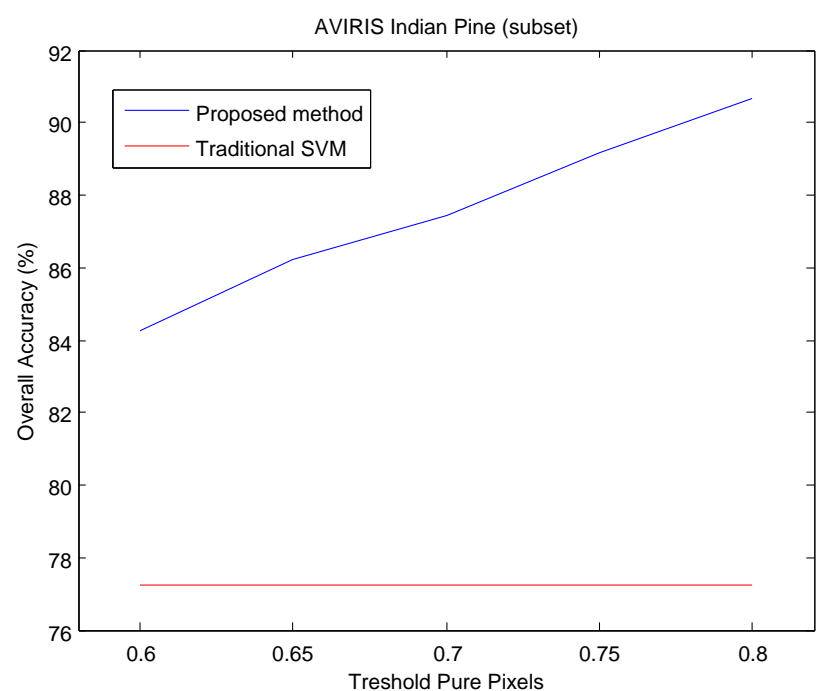

(a)

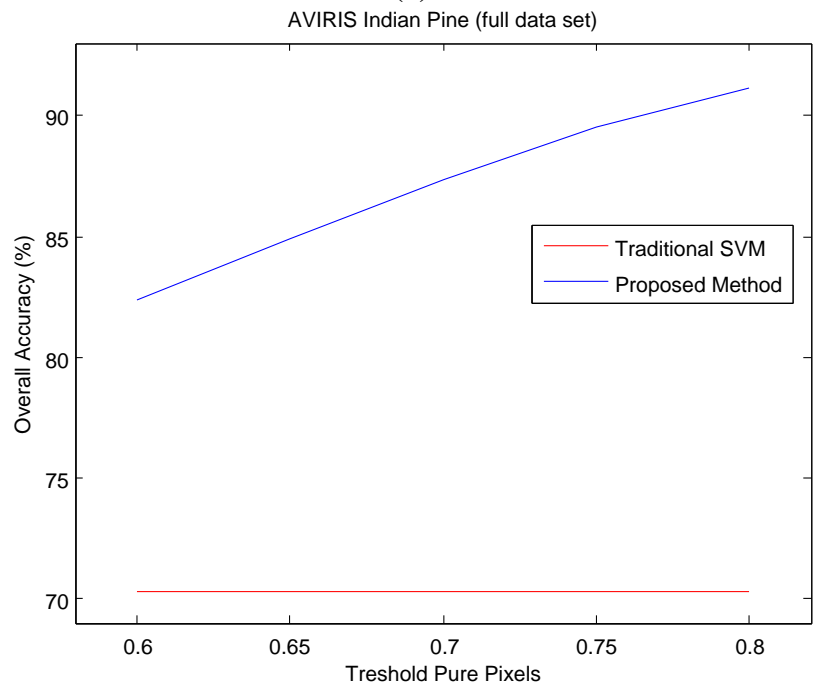

(c)

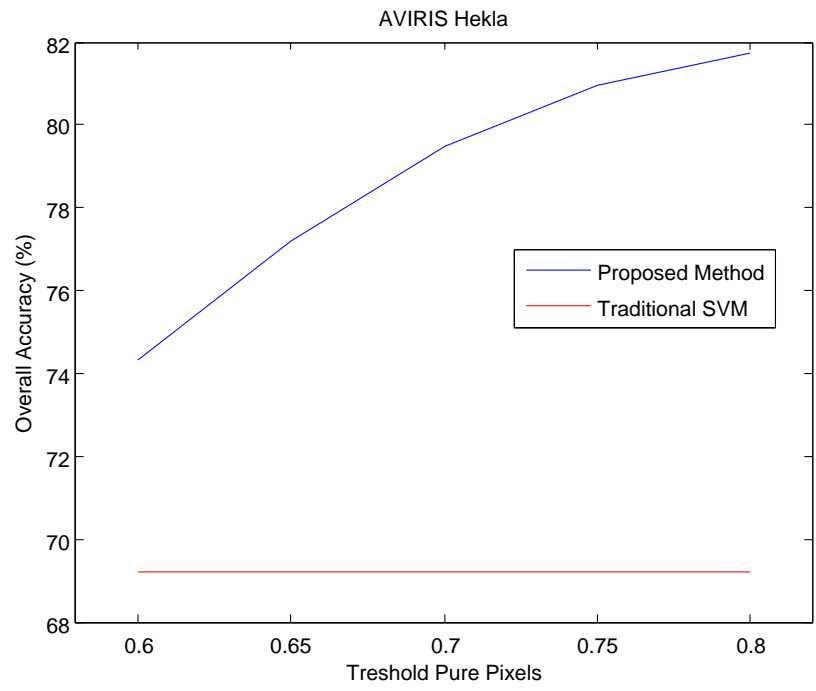

(e)

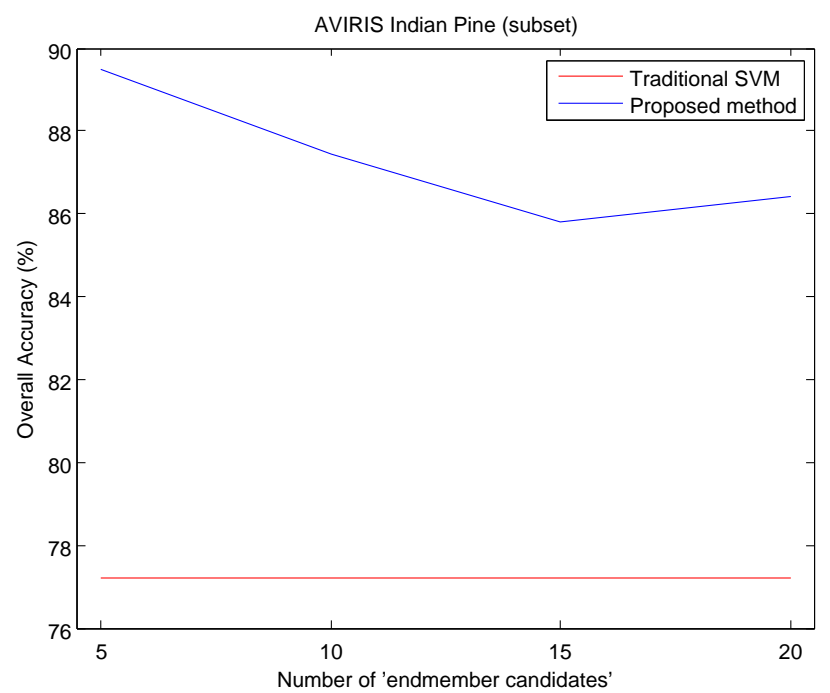

(b)

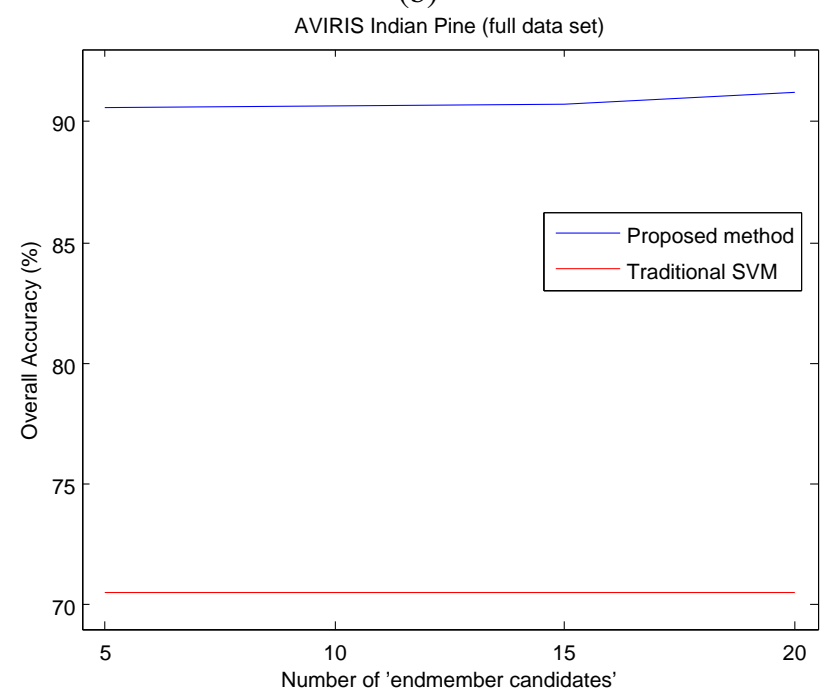

(d)

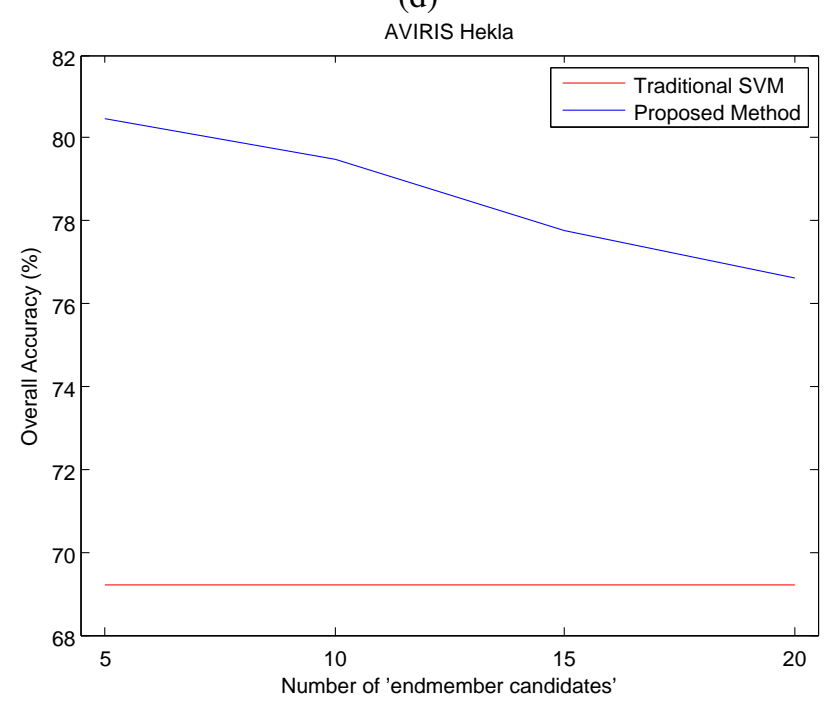

(f)

Fig. 8. Variation of the overall classification accuracy versus the value of the parameter treshold to determine if a pixel can be considered as 'pure' for (a) AVIRIS Indian Pine subset (b) AVIRIS Indian Pine complete (c) AVIRIS Hekla data sets

Copyright (c) 2010 IEEE. Personal use is permitted. For any other purposes, Permission must be obtained from the IEEE by emailing pubs-permissions@ieee.org. 
TABLE V

COMPUTATIONAL BURDEN FOR THE THREE DATA SETS.

\begin{tabular}{|c|c|c|c|c|c|c|}
\hline & \multicolumn{2}{|c|}{ AVIRIS Indian Pine subset } & AVIRIS Indian Pine complete & \multicolumn{2}{c|}{ AVIRIS Hekla } \\
\hline Approach & SVM & Proposed Method & SVM & Proposed Method & SVM & Proposed Method \\
\hline Classification & $30 \mathrm{~s}$ & $43 \mathrm{~s}$ & $14 \mathrm{~min}$ & $15 \mathrm{~min}$ & $35 \mathrm{~min}$ & $37 \mathrm{~min}$ \\
Spectral Unmixing & - & $15 \mathrm{~s}$ & - & $1.5 \mathrm{~min}$ & - & $2 \mathrm{~min}$ \\
Simulated Annealing & - & $50 \mathrm{~s}$ & - & $3 \mathrm{~min}$ & - & $4 \mathrm{~min}$ \\
\hline Total elapsed time & $30 \mathrm{~s}$ & $88 \mathrm{~s}$ & $18 \mathrm{~min}$ & $23 \mathrm{~min}$ & $35 \mathrm{~min}$ & $43 \mathrm{~min}$ \\
\hline
\end{tabular}

the obtained classification maps are shown in Fig. $7.15 \%$ of the labeled pixels of the low resolution data were randomly selected from each class and used to train the classifier, and the experiment repeated ten times with different training sets. The quantitative results in this experiment confirm those obtained in the previous ones: the proposed method provides not only a better classification map from a qualitative point of view, but also a large improvement of the overall accuracy of correctly classified pixels. Due to the irregular spatial structures in which the land cover classes are grouped, the spatial regularization method proposed in this paper was expected to be less effective than in the previous cases. The quantitative results agree with this supposition, also if the overall accuracy is penalized by only 2 percentage points. More advanced techniques could be investigated in our future works, in order to have an improvement of the classification accuracy of the mixed pixels.

\section{Discussion about the choice of parameters and computa- tional burden}

In the proposed method, the parameters having an influence on the overall classification accuracy obtained (apart from the parameters of the SVM, which are automatically selected through cross-validation) are the treshold to distinguish between pure and mixed pixels and the number of 'endmember candidates' to consider for the spectral unmixing, in the second step. How the classification accuracy changes by changing the value of the parameters can be seen in Fig. IV-B. It can be noticed that the proposed method outperforms the traditional SVM in terms of accuracy over the whole range tested, being the choice of the parameters not crucial for the classification. As could be expected, a high value of the treshold to determine if a pixel can be considered as 'pure' provides a higher accuracy, since only the most reliable pixels are labeled for the preliminary classification. By setting a low value of the treshold parameter, the preliminary classification map will tend to be like the hard classification map obtained with a traditional SVM, thus decreasing the interest of the proposed method.

The number of 'endmember candidates' considered in the spectral unmixing step points out the importance of the spatial information. The best results are in general obtained by considering a low number of candidates (which are the spatially closest to the considered pixel). When setting a larger value of the parameter, endmember candidates spatially far from the analysed pixel can be selected, introducing useless information and thus leading to a slight decrease in the classification accuracy.
The computational burden of the proposed method can be seen in Table V. The training of the SVM, which quadratically depends on the size of the training set, is the most computationally expensive step of the proposed approach. The spectral unmixing step depends on the number of the mixed pixel to unmix, while the Simulated Annealing regularization depends on the number of mixed pixels and on the zoom factor desired. When requiring a larger zoom, the number of possible sub-pixel combinations grows exponentially, thus requiring a heavier computational burden to reach the optimal configuration. In case of desired zoom factor equal or higher than 4 , the computational burden of the spatial regularization is expected the be the most important.

\section{CONCLusions}

Classification of hyperspectral images in presence of mixed pixels was addressed in this paper. A new method for the improvement of the spatial resolution of the classification maps was proposed. The method exploits the advantages of both soft classification techniques and spectral unmixing algorithms, in order to determine the fractional abundances of the classes at a sub-pixel scale. After the fractional abundances have been determined, spatial regularization by Simulated Annealing is finally performed to spatially locate the land cover classes within each pixel. Experiments were carried out on three different data sets and show that the proposed method clearly outperforms classical classification techniques when areas with mixtures of materials are located in the scene, providing excellent results both from a visually and quantitative point of view. Further research will be devoted to the investigation of advanced methods to better discriminate pure and mixed pixels, and of the possibility of alternative techniques of spatial regularization.

Acknowledgments: This work has been supported by the European Community's Marie Curie Research Training Networks Programme under contract MRTN-CT-2006-035927, Hyperspectral Imaging Network (HYPER-I-NET).

\section{REFERENCES}

[1] D. A. Landgrebe, Signal Theory Methods in Multispectral Remote Sensing. John Wiley and Sons, 2003.

[2] G. Vane, R. Green, T. Chrien, H. Enmark, E. Hansen, and W. Porter, "The airborne visible/infrared imaging spectrometer (aviris)," Remote Sens. Environ., vol. 44, pp. 127-143, 1993.

[3] A. Cracknell, "Synergy in remote sensing: What's in a pixel?" Int. J. Remote Sens., vol. 19, pp. 2025-2047, 1998.

[4] G. Camps-Valls and L. B. (eds.), Kernel methods for Remote Sensing Data Analysis. John Wiley and Sons, 2009.

[5] M. Nachtegael, D. Weken, E. van der Kerre, and W. Philips, Eds., Soft Computing in Image Processing. Springer, 2007. 
[6] N. Keshava and J. F. Mustard, "Spectral unmixing," IEEE Signal Proc. Mag., vol. 19, pp. 44-57, 2002.

[7] A. Plaza, P. Martinez, R. Perez, , and J. Plaza, "A quantitative and comparative analysis of endmember extraction algorithms from hyperspectral data," IEEE Trans. Geosci. Remote Sens., vol. 42, no. 3, pp. 650-653, Mar. 2004.

[8] A. Plaza, G. Martin, J. Plaza, M. Zortea, and S. Sanchez, in Optical Remote Sensing - Advances in Signal Processing and Exploitation Techniques. Springer, 2010, ch. ”Recent Developments in Spectral Unmixing and Endmember Extraction".

[9] J. Li and L. M. Bruce, "Wavelet-based feature extraction for improved endmember abundance estimation in linear unmixing of hyperspectral signals," IEEE Trans. Geosci. Remote Sens., vol. 42, no. 3, pp. 644-649, Mar. 2004.

[10] L. Wang and X. Jia, "Integration of soft and hard classifications using extended support vector machines," IEEE Geosci. Remote Sens. Letters, vol. 6, no. 3, pp. 543-547, Jul. 2009.

[11] A. Plaza, P. Martinez, R. Perez, and J. Plaza, "A new approach to mixed pixel classification of hyperspectral imagery based on extended morphological profiles," Pattern Recognition, vol. 37, pp. 1097-1116, 2004.

[12] P. Atkinson, Innovations in GIS. New York Taylor and Francis, 1997, vol. 4, ch. Mapping sub-pixel boundaries from remotely sensed images, pp. $166-180$.

[13] K. Mertens, "Sub-pixel mapping: A comparison of techniques," Ph.D. dissertation, Ghent University, 2008.

[14] A. Bardossy and L. Samaniego, "Fuzzy-rule based classification of remotely sensed imagery," IEEE Trans. Geosci. Remote Sens., vol. 2, no. 2, pp. 362-274, 2002.

[15] A. Robin, S. L. Hegarat-Mascle, and L. Moisan, "Unsupervised subpixelic classification using coarse-resolution time series and structural information," IEEE Trans. Geosci. Remote Sens., vol. 46, no. 5, pp. 1359-1374, May 2008.

[16] P. Debba, E. Carranza, F. van der Meer, and A. Stein, "Abundances estimation of spectrally similar minerals by using derivative spectra in simulated annealing," IEEE Trans. Geosci. Remote Sens., vol. 44, no. 12, pp. 3469-3658, Dec. 2006.

[17] V. Vapnik, Statistical Learning Theory. New York: Wiley, 1998.

[18] F. Melgani and L. Bruzzone, "Classification of hyperspectral remote sensing images with support vector machine," IEEE Trans. Geosci. Remote Sens., vol. 42, no. 8, pp. 1778-1790, Aug. 2004.

[19] G. Licciardi, F. Pacifici, D. Tuia, S. Prasad, T. West, F. Giacco, C. Thiel, J. Inglada, E. Christophe, J. Chanussot, and P. Gamba, "Decision fusion for the classification of hyperspectral data: Outcome of the 2008 grs-s data fusion contest," IEEE Trans. Geosci. Remote Sens., vol. 47, pp. 3587-3865, 2009.

[20] M. Fauvel, J. Chanussot, J. Benediktsson, and J. Sveinsson, "Spectral and spatial classification of hyperspectral data using svms and morphological profiles," IEEE Trans. Geosci. Remote Sens., vol. 46, no. 10, pp. 3804-3814, Oct. 2008.

[21] N. Aronszajn, "Theory of reprodusing kernel," Harvard University, Division of engineering sciences, Tech. Rep., 1950.

[22] J. Platt, Advances in Large Margin Classifiers. Cambridge, MA: MIT Press, 2000, ch. Probabilistic outputs for support vector machines and comparison to regularized likelihood methods.

[23] H. Lin, C. Lin, and R. C. Weng, "A note on platt's probabilistic outputs for support vector machines," Dept. Comput. Sci., Nat. Taiwan Univ., Taipei, Taiwan, Tech. Rep., 2003.

[24] C. Chang and C. Lin, "Libsvm: A library for support vector machines," 2001, [Online]. Available: http://www.csie.ntu.edu.tw/ cjlin/libsvm.

[25] G. Foody and H. Doan, "Variability in soft classification prediction and its implications for sub-pixel scale change detection and super-resolution mapping," Photogramm. Eng. Remote Sens., vol. 73, pp. 923-933, 2007.

[26] D. Heinz and C. Chang, "Fully constrained least squares linear spectral mixture analysis method for material quantification in hyperspectral imagery," IEEE Trans. Geosci. Remote Sens., vol. 39, no. 3, pp. 529545, Mar. 2001

[27] Z. Kulpa, "More about areas and perimeters of quantized objects," Computer Vision, Graphics, and Image Processing, vol. 22, no. 2, pp. 268-276, May 1983.

[28] N. Metropolis, A. Rosenbluth, M. Rosenbluth, A. Teller, and E. Teller, "Equations of state calculations by fast computing machines," J. Chem. Phys., vol. 21, pp. 1087-1092, 1958.

[29] S. Kirkpatrick, C. Gelatt, and M. Vecchi, "Optimization by simulated annealing," Science, vol. 220, pp. 671-680, 1983.
[30] R. N. Clark, G. A. Swayze, R. Wise, K. E. Livo, T. M. Hoefen, R. F. Kokaly, and S. J. Sutley, "Usgs digital spectral library splib06a," U.S. Geological Survey, Data Series 231, 2007, available on-line at: http://speclab.cr.usgs.gov/spectral.lib06/ds231/datatable.html.

[31] C. Hsu and C. Lin, "A comparison of methods for multi-class support vector machines," IEEE Trans. Neural Networks, vol. 13, no. 2, pp. 415-425, 2002.

[32] J. Cohen, "A coefficient of agreement for nominal scales," Educational and psychological measurement, vol. 46, pp. 20-37, 1960.

[33] J. Gualtieri, S. Chettri, R. Cromp, and L. Johnson, "Support vector machine classifiers as applied to aviris data," in Proc. 8th JPL Airborne Geosci. Workshop, Feb. 1999, pp. 217-227.

[34] Y. Tarabalka, J. Chanussot, and J. A. Benediktsson, "Segmentation and classification of hyperspectral images using minimum spanning forest grown from automatically selected markers," IEEE Trans. on Systems, Man, and Cybernetics, Part B: Cybernetics, vol. 40, no. 5, pp. 12671279, 2010.

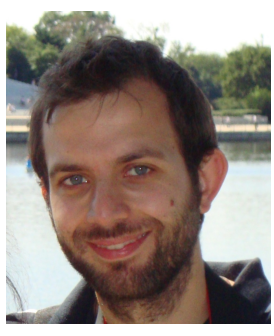

Alberto Villa (S'09) received the B.S. and M.S. degrees in electronic engineering from the University of Pavia, Italy, in 2005 and 2008, respectively. Since 2008, he has been working toward the Ph.D. degree, a joint degree between the Grenoble Institute of Technology (Grenoble INP), Grenoble, France and the University of Iceland, Reykjavik, Iceland. His research interests are in the areas of spectral unmixing, machine learning, hyperspectral imaging, signal and image processing.

$\mathrm{He}$ is a Reviewer for IEEE TRANSACTIONS ON GEOSCIENCE AND REMOTE SENSING, IEEE JOURNAL OF SELECTED TOPIC IN SIGNAL PROCESSING and JOURNAL OF SIGNAL PROCESSING SYSTEMS.

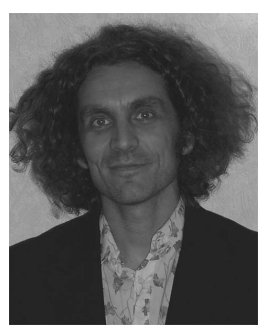

Jocelyn Chanussot (M'04-SM'04) received the M.Sc. degree in electrical engineering from the Grenoble Institute of Technology (Grenoble INP), Grenoble, France, in 1995 and the Ph.D. degree from the University of Savoie, Annecy, France, in 1998. In 1999, he was with the Geography Imagery Perception Laboratory, Délégation Genérale pour l'Armement (French National Defense Department). Since 1999, he has been with Grenoble INP, where he was an Assistant Professor from 1999 to 2005, was an Associate Professor from 2005 to 2007, and is currently a Professor of signal and image processing. He is currently conducting his research at the Grenoble Images Speech Signals and Automatics Laboratory, (GIPSA-Lab, affiliated to CNRS and Grenoble INP). His research interests include image analysis, multicomponent image processing, nonlinear filtering, and data fusion in remote sensing.

Dr. Chanussot is the founding President of the IEEE Geoscience and Remote Sensing French Chapter (2007) which was the recipient of the 2010 IEEE GRSS Chapter Excellence Award for excellence as a Geoscience and Remote Sensing Society chapter demonstrated by exemplary activities during 2009 . $\mathrm{He}$ is a member of the IEEE Geoscience and Remote Sensing AdCom (20092011). He was the General Chair of the first IEEE GRSS Workshop on Hyperspectral Image and Signal Processing-Evolution in Remote Sensing (2008) and the technical co-chair of the following editions. He is the Chair (2009-2011) and was the Cochair (2005-2008) of the GRS-S Data Fusion Technical Committee. He was a member of the Machine Learning for Signal Processing Technical Committee of the IEEE Signal Processing Society (20062008) and the Program Chair of the IEEE International Workshop on Machine Learning for Signal Processing (2009). He was an Associate Editor for the IEEE GEOSCIENCE AND REMOTE SENSING LETTERS (2005-2007) and for Pattern Recognition (2006-2008). He has been an Associate Editor for the IEEE TRANSACTIONS ON GEOSCIENCE AND REMOTE SENSING since 2007. He is currently the Editor in Chief of the IEEE Journal of Selected Topics in Applied Earth Observations and Remote Sensing (J-STARS) (20112013). 


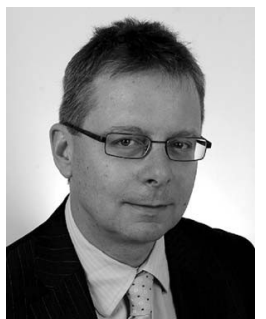

Jón Atli Benediktsson (S'84-M'90-SM'99-F'04) received the Cand.Sci. degree in electrical engineering from the University of Iceland, Reykjavik, in 1984, and the M.S.E.E. and Ph.D. degrees from Purdue University, West Lafayette, IN, in 1987 and 1990, respectively. He is currently Pro Rector for Academic Affairs and Professor of Electrical and Computer Engineering at the University of Iceland. His research interests are in remote sensing, biomedical analysis of singals, pattern recognition, image processing, and signal processing, and he has published extensively in those fields. Prof. Benediktsson is the 2011 President of the IEEE Geoscience and and Remote Sensing Society (GRSS) but he has been on the GRSS AdCom since 2000. He was Editor of the IEEE Transactions on Geoscience and Remote Sensing (TGARS) from 2003 to 2008. He received the Stevan J. Kristof Award from Purdue University in 1991 as outstanding graduate student in remote sensing. In 1997, Dr. Benediktsson was the recipient of the Icelandic Research Council's Outstanding Young Researcher Award, in 2000, he was granted the IEEE Third Millennium Medal, in 2004, he was a co-recipient of the University of Iceland's Technology Innovation Award, in 2006 he received the yearly research award from the Engineering Research Institute of the University of Iceland, and in 2007, he received the Outstanding Service Award from the IEEE Geoscience and Remote Sensing Society. He is a member of Societas Scinetiarum Islandica and Tau Beta Pi.

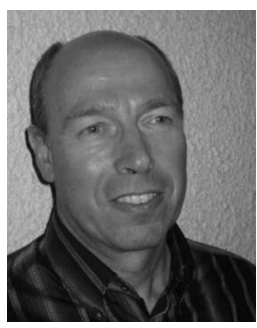

Christian Jutten (M'03-SM'06-F'08) received the $\mathrm{PhD}$ degree in 1981 and the Docteur des Sciences degree in 1987 from the Institut National Polytechnique of Grenoble (France). After being associate professor in the Electrical Engineering Department in the Institut National Polytechnique of Grenoble (1982-1989) and visiting professor in Swiss Federal Polytechnic Institute in Lausanne (1989), he became full professor in University Joseph Fourier of Grenoble, more precisely in the sciences and technologies department. For 30 years, his research interests are learning in neural networks, blind source separation and independent component analysis, including theoretical aspects (separability, source separation in nonlinear mixtures) and applications (biomedical, seismic, speech, astrophysics, chemical sensor array).

$\mathrm{He}$ is author or co-author of 4 books, 18 invited papers, and more than 60 papers in international journals and 160 communications in international conferences. He was co-organizer of the 1 st International Conference on Blind Signal Separation and Independent Component Analysis (Aussois, France, January 1999). He has been a scientific advisor for signal and images processing at the French Ministry of Research (1996-1998) and for the French National Research Center (CNRS, 2003-2006). He has been associate editor of IEEE Trans. on Circuits and Systems (1994-95). He is a member of the technical committee Blind Signal Processing of the IEEE CAS society and of the technical committee Machine Learning for Signal Processing of the IEEE SP society. For his contributions in source separation and independent component analysis, he received the EURASIP best paper award in 1992, Medal Blondel in 1997 from SEE (French Electrical Engineering society) and, in 2008, has been elevated as a Fellow IEEE and a senior Member of Institut Universitaire de France. 